\title{
Atypical cases of filariasis from a non-endemic area
}

\author{
Parul Kodan ${ }^{1}$, Nitin Gupta ${ }^{1}$, Ankita Baidya ${ }^{1}$, Ayan Basu ${ }^{1}$, Abdul Razik ${ }^{2}$, Ankit Mittal ${ }^{1}$, \\ Nishant Verma ${ }^{3}$, Bijay Ranjan Mirdha ${ }^{3}$, Sandeep Mathur ${ }^{4}$, Madhu Rajeshwari ${ }^{4}$, \\ Neeraj Nischal ${ }^{5}$, Manish Soneja, ${ }^{5, *}$, Naveet Wig ${ }^{5}$ \\ ${ }^{1}$ Department of Medicine and Microbiology, All India Institute of Medical Sciences, New Delhi, India; \\ ${ }^{2}$ Department of Radiodiagnosis, All India Institute of Medical Sciences, New Delhi, India; \\ ${ }^{3}$ Department of Microbiology, All India Institute of Medical Sciences, New Delhi, India; \\ ${ }^{4}$ Department of Pathology, All India Institute of Medical Sciences, New Delhi, India; \\ ${ }^{5}$ Department of Medicine, All India Institute of Medical Sciences, New Delhi, India.
}

\begin{abstract}
Summary Filariasis can present in many different ways and pose significant dilemma to the clinician. We report four atypical cases of filariasis which presented as abdominal mass, cervical lymph node enlargement, fever in pregnancy and nosocomial febrile illness respectively. All the four cases were treated successfully with oral antifilarial agents. It is essential to be aware of such atypical presentations of filariasis so that prompt therapy can be initiated.
\end{abstract}

Keywords: Wuchereria bancrofti, pregnancy, lymphadenopathy

\section{Introduction}

Filariasis is a neglected tropical disease which poses a significant burden in endemic areas. An estimated 68 million people are infected worldwide, with 40 million being symptomatic $(1,2)$. India shares a heavy burden of the disease and despite having a successful national program, it is not rare to find people from endemic areas presenting with swollen limbs and scrotal swelling even today (3). The social, psychological and economic implications of this disease are enormous. Unfortunately, the disease is often missed in the early stages and when it manifests atypically. It can present in many different ways including fever, lymphangitis, cough or wheeze and limb or scrotal swelling. Since it may be incidentally detected in the blood or tissue aspirate, it is imperative for the laboratory technicians and clinicians to be aware of such findings. Timely diagnosis and appropriate management can significantly decrease the morbidity associated with the disease. We present four atypical cases of filariasis which created significant diagnostic dilemmas but were successfully managed.

\footnotetext{
*Address correspondence to:

Dr. Manish Soneja, Department of Medicine, 3rd floor, Teaching block, All India Institute of Medical Sciences, New Delhi, India.

E-mail:manishsoneja@gmail.com
}

\section{Case Report}

\subsection{Case 1: Filariasis mimicking a malignant abdominal tumor}

A forty-eight-year old female, resident of Uttar Pradesh, presented with a lump in the right lumbar area, which developed over a year. Her blood investigations were normal. Magnetic Resonance Imaging (MRI) of the abdomen revealed a large midline, complex cystic mass of size $8.5 \mathrm{~cm}$ in the greater pelvis showing fluid-debris level as well as non-dependent membranous structures within (Figure 1A). The ovaries were seen separately from the mass. There was another complex cystic mass of size 5 $\mathrm{cm}$ in the right pararenal space of retroperitoneum, inferior to the right kidney (Figure 1B). Some of the loculi showed T2-hypointense debris. In addition, conglomerate tubular channels were seen along the bilateral external and internal iliac vessels, which raised the suspicion of dilated lymphatics (Figure 1C). The magnetic resonance lymphangiogram subsequently performed confirmed the lymphatic nature of these tubular channels. The cystic lesions in the pelvis and retroperitoneum also showed partial opacification with gadolinium, suggesting lymphatic communication and hence the origin of these cysts. Imaging differentials of multifocal macrocystic lymphatic malformation as well as of 
cystic mesothelial and germ cell neoplasms were kept. Since imaging could not rule out malignancy, fine needle aspiration cytology (FNAC) of the pelvic mass was done, which revealed microfilaria (Figure 2). She was treated with oral diethycarbazine (DEC) for twenty-one days, subsequent to which the abdominal lump gradually decreased in size. The follow-up MRI performed at one year showed complete resolution of the pelvic and retroperitoneal lumps as well as the lymphatic dilatation (Figure 1D).

\subsection{Case 2: Microfilariae in the lymph node}

A eleven-year-old female, resident of Delhi, presented with on and off fever with chills for six months. On physical examination, she was found to have an enlarged right cervical lymph node measuring $4 \times 2 \mathrm{~cm}$. Her routine blood investigations were normal. Mantoux test was negative. Cytopathology of the lymph node aspirate revealed reactive changes with presence of microfilariae. She was treated with DEC and
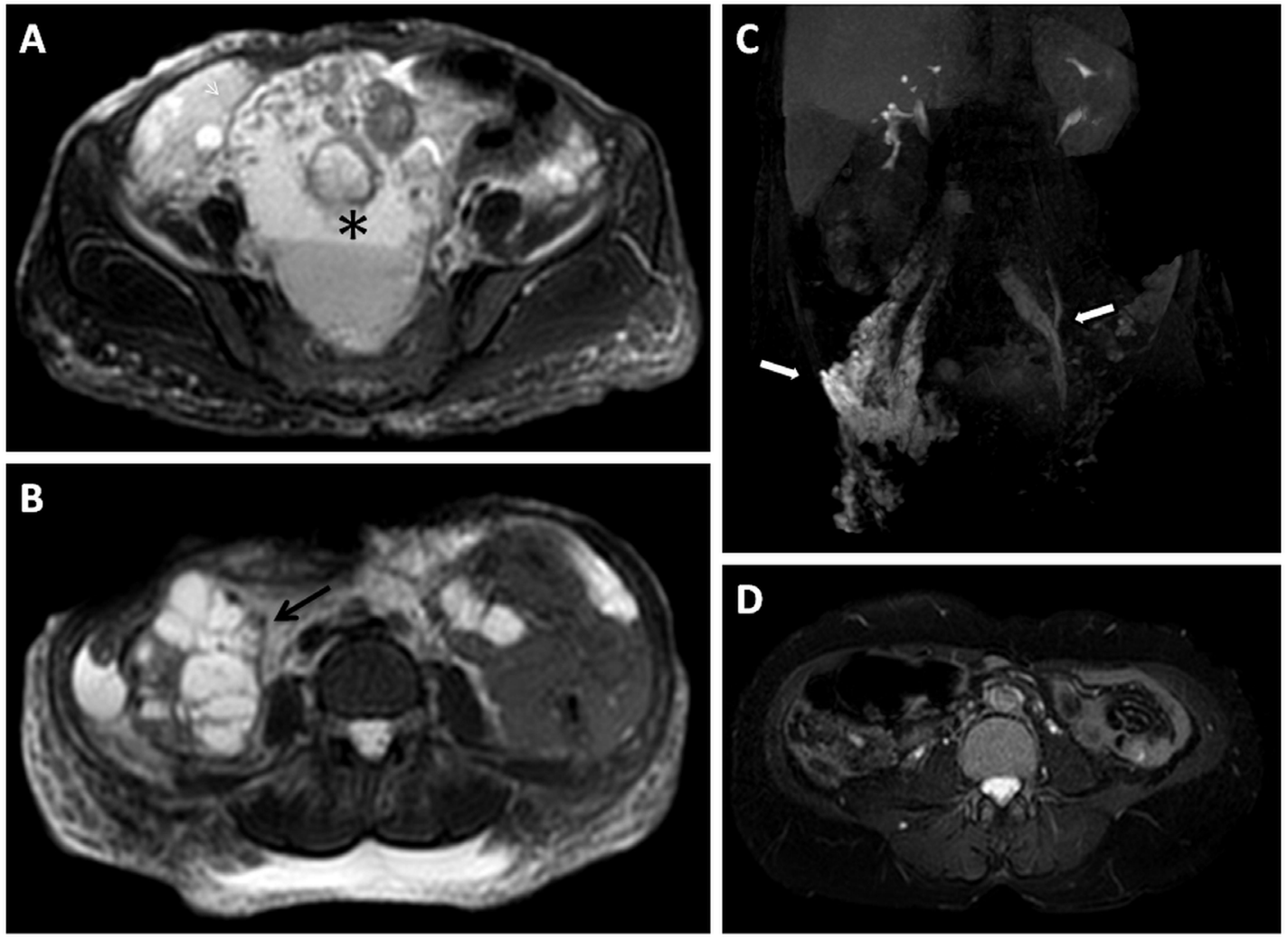

Figure 1. Fat suppressed T2-weighted MRI showing (A) large complex pelvic cystic mass (asterisk) with fluid-debris level as well as numerous T2-hypointense membranous contents, and (B) a complex multiseptate cyst (arrow) in the retroperitoneum on the right side. Some of the loculi show differential T2-hypointensity, consistent with debris. (C) Coronal maximum intensity projection images of MR lymphangiogram showing dilated lymphatics (open arrows) along the bilateral external and common iliac vessels. (D) Representative post-treatment T2-weighted MRI at the level of the second image showing complete resolution after one year of therapy.
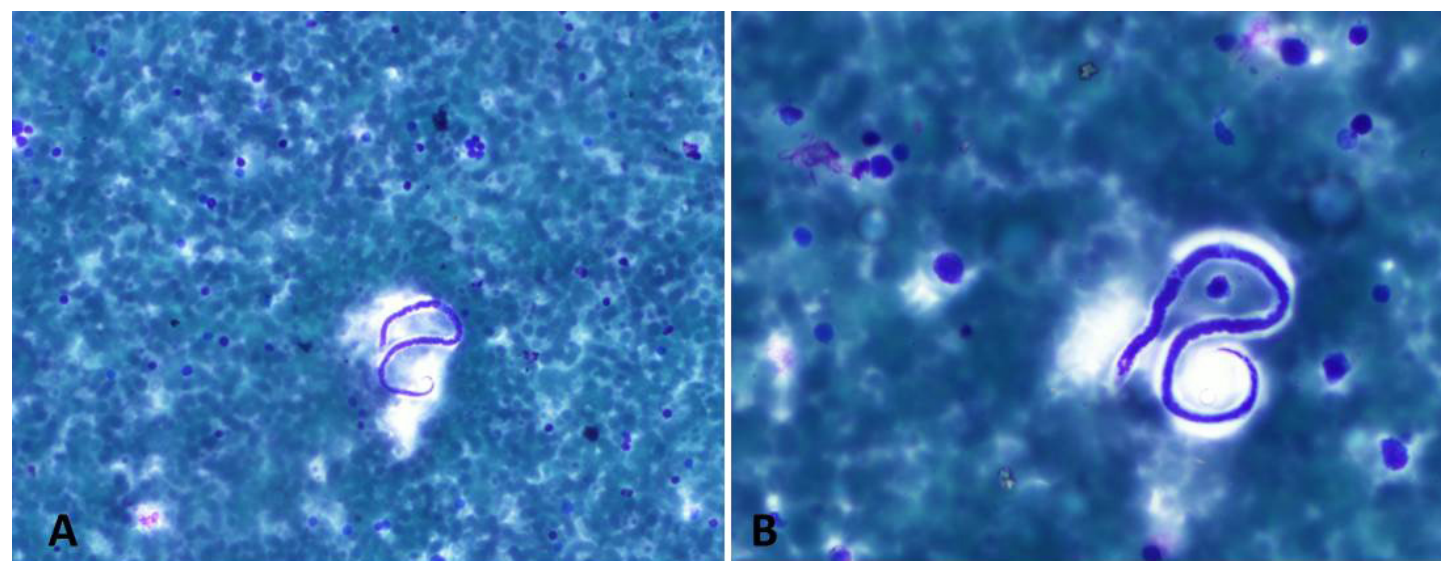

Figure 2. Fine needle aspiration smear from abdominal lump showing microfilaria in a background of blood and inflammatory cells (A). A higher power view of the microfilaria surrounded by a faintly staining sheath (B). 
doxycycline, subsequent to which the fever resolved and did not recur at four months of follow. However, the lymph node swelling was persistent.

\subsection{Case 3: Detection of microfilariae in the screening peripheral smear of a pregnant patient with fever}

A 24-year-old pregnant female, resident of Delhi, presented in the second trimester of her pregnancy with fever, chills and joint pain lasting three days. The physical examination was unremarkable. Her routine peripheral blood smear showed microfilariae. Oral albendazole was initiated and the patient became afebrile on the second day of treatment. She remained asymptomatic at six months of follow-up and no microfilariae were seen in follow-up smear.

\subsection{Case 4: From the postoperative ward for fever}

A 55-year-old hypertensive male from Delhi presented acutely with altered sensorium and hemiparesis. Unenhanced computed tomography (CT) of the brain showed acute bleed in the left basal ganglion with midline shift and intraventricular extension. He underwent craniotomy with hematoma evacuation and frontal external ventricular drain (EVD) insertion. On the seventh postoperative day, he developed high-grade fever with chills. Physical examination revealed no focus of infection. He was started on broad-spectrum antibiotics, however there was no improvement in the fever. His blood counts, liver and kidney function tests, blood and urine cultures, cerebrospinal fluid

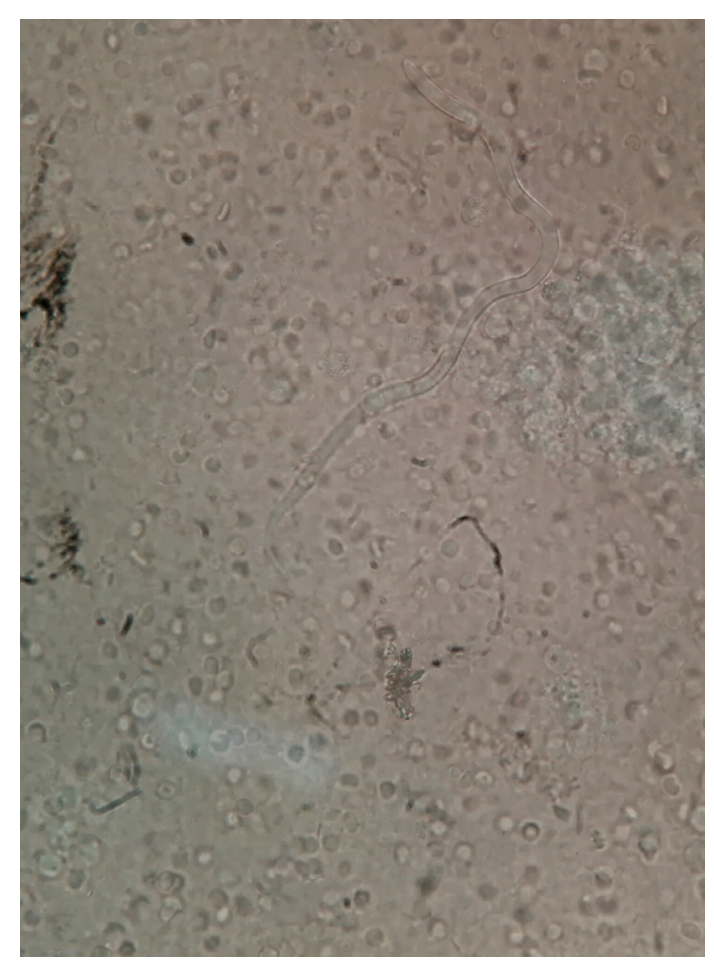

Figure 3. Wet mount of blood showing microfilaria. examination, serum procalcitonin levels and chest radiograph were normal. Blood mount showed motile microfilariae (Figure 3), which were identified as Wuchereria bancrofti on giemsa staining (Figure 4). He was started on oral DEC and became afebrile on the second day of treatment.

\section{Discussion}

Filariasis is a major public health problem in the tropical world with both lymphatic and extralymphatic manifestations. A retrospective analysis of the records of our outpatient clinics and infectious disease consultations from 2017 revealed 20 patients of proven filariasis. Five patients showed atypical presentations, with the findings of one among them (massive splenomegaly with bone marrow aspirate showing microfilariae) having been already published (4).

Microfilariae have been detected incidentally in unsuspected swellings in many reports from across the world (5-8). In such cases, microfilariae may either be the direct etiologic agent for the swelling or may have gotten entrapped in an already existent swelling. Diagnosing extralymphatic filariasis remains a clinical challenge as it is often unsuspected and as it mimics non-filarial diseases. Our series describes four cases of filariasis which presented at unusual and rare sites, but were promptly diagnosed and managed.

The first case showed complex cystic masses in the pelvis and retroperitoneum, which usually raise the suspicion of macrocystic lymphatic malformation or malignancy. Since the ovaries were separately visualized, the possible differentials included primary cystic mesothelial and germ cell neoplasms. Because of the unusual morphology, filariasis was not kept as a differential until the cytology revealed otherwise. Timely diagnosis and prompt institution of antihelminthic therapy enabled complete recovery

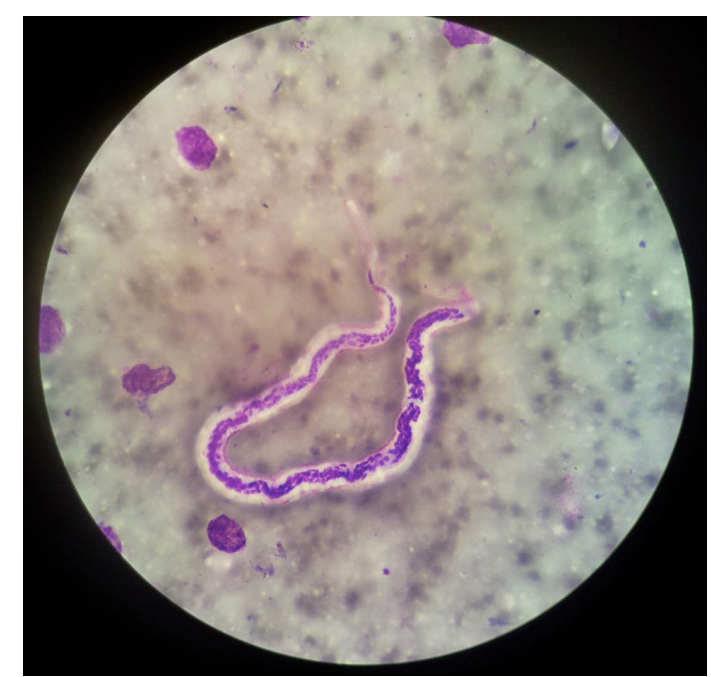

Figure 4. Peripheral blood smear (Giemsa stain) showing microfilaria of Wuchereria bancrofti. 
without surgery. Only four reports of filariasis presenting as abdominal masses or cysts are available in the current literature, all of which are from the Indian subcontinent (9-12). Except for one, all showed large unilocular retroperitoneal cysts which were resected. Giri et al. (12) reported a complex solidcystic retroperitoneal mass which, similar to our case, resolved completely on oral DEC therapy. Although the exact mechanism of the origin of abdominal cysts in filariasis is not known, possible hypothesis includes lymphatic obstruction resulting in rupture and extravasation of chyle as well as the involvement of ectopic lymphatic tissue (9). Although rare, keeping a high index of suspicion can result in timely diagnosis, which avoids surgery and significantly changes the course of the disease.

Many case series and reports have shown incidental detection of microfilaria in lymph node aspirates (6-8). Presence of microfilariae in lymph nodes may only be an incidental finding with no proven causal association. Cases of microfilariae in aspirates from malignant lymph nodes have also been reported, although the association with malignancy is controversial (13-17). It is important to be aware of this observation and so that the samples are carefully screened. Timely diagnosis can prevent future morbidity and complications.

There is no definitive evidence as to whether pregnancy, surgery or other forms of stress can activate the dormant worm and release more microfilariae into blood. No cases of filariasis presenting as nosocomial fever has been described in the literature. However, two among the four cases in our series presented for the first time in periods of stress (pregnancy and postoperative period respectively). Whether these presentations were incidental or had any possible relation to stress remains speculative. Another noteworthy finding in our case series was that none of the patients had eosinophilia. This highlights the fact that eosinophil counts may be normal in filariasis and the diagnosis should not be ruled out for the mere absence of eosinophilia.

There have been several reports of filariasis from non-endemic areas such as Delhi. Two among the four patients belonged to Delhi and had no history of travel to other endemic areas. Literature suggests that transmission of filariasis requires prolonged stay in endemic areas and hence is uncommon in travelers.

Detection of microfilaria or adult worm in the laboratory samples should prompt the physician to initiate treatment so as to avoid any long-term consequences of the disease. DEC is highly effective in eliminating microfilaria, but has only modest activity against the adult worm. Many recent studies support the synergistic effect of combining this drug with doxycycline. Doxycycline acts by targeting Wolbachia, which engages in endosymbiotic relationship with the filarial parasite and is important in maintaining the reproductive capacity of the worm (18-20). Albendazole and ivermectin have been used in combination as alternatives. Ivermectin and doxycycline should be avoided in pregnancy. In our series, all the patients responded well to treatment with oral anti-filarial drugs (DEC or albendazole). All patients tolerated the drug well and did not report any side effects or intolerance. All patients showed improvement in symptoms at follow up.

As we envision a world free of filariasis, the World Health Organization (WHO) has launched the Global Programme to Eliminate Lymphatic Filariasis (GPELF) by 2020 . WHO recommends preventive chemotherapy with annual mass drug administration (MDA) of albendazole together with ivermectin or with DEC. Enhanced public health strategies, disease awareness, prompt diagnosis and treatment can help us in paving the path towards elimination.

In conclusion, these cases highlight the unusual and diagnostically challenging presentations of filariasis. It underscores the fact that timely diagnosis and prompt management can prevent severe complications and long-term sequelae. Each patient who gets diagnosed and treated moves us a step closer towards the vision of filariasis-free world.

\section{References}

1. Ramaiah KD, Ottesen EA. Progress and impact of 13 years of the global programme to eliminate lymphatic filariasis on reducing the burden of filarialdisease. PLoS Negl Trop Dis. 2014; 8:e3319.

2. Rebollo MP, Bockarie MJ. Toward the elimination of lymphatic filariasis by2020: Treatment update and impact assessment for the endgame. Expert Rev AntiInfect Ther. 2013; 11:723-731.

3. Raju K, Jambulingam P, Sabesan S, Vanamail P. Lymphatic filariasis in India:epidemiology and control measures. J Postgrad Med. 2010; 56:232-238.

4. Basu A, Kumar A, Manchanda S, Wig N. Filarial huge splenomegaly dramatically regressed by anti-filarial medication: A rare clinical scenario. Intractable Rare Dis Res. 2017; 6:215-218.

5. Sah SP, Rani S, Mahto R. Microfilariae in lymph node aspirates. Acta Cytol. 2002; 46:73-75.

6. Varghese R, Raghuveer CV, Pai MR, Bansal R. Microfilariae in cytologicsmears: A report of six cases. Acta Cytol. 1996; 40:299-301.

7. Sivakumar S. Role of fine needle aspiration cytology in detection ofmicrofilariae: Report of 2 cases. Acta Cytol. 2007; 51:803-806.

8. Mitra SK, Mishra RK, Verma P. Cytological diagnosis of microfilariae in filariasis endemic areas of eastern Uttar Pradesh. J Cytol. 2009; 26:11-14.

9. Kapoor AK, Puri SK, Arora A, Upreti L, Puri AS. Case report: Filariasis presenting as an intra-abdominal cyst. Indian J Radiol Imaging. 2011; 21:18-20.

10. Pillay CR. Retroperitoneal cyst of filarial origin. J Assoc Physicians India. 1961; 9:760-762.

11. Mehta RB, Gajendran V, Ananthakrishnan N, Parkash S. Retroperitoneal tumorous lesions. Indian J Surg. 1981; 43:731-742. 
12. Giri A, Kundu AK, Chakraborty M, Das S. Microfilarial worms in retroperitoneal mass: A case report. Indian J Urol. 2000; 17:57-58.

13. Yadav R, Sagar M, Maurya M.K, A. A Sonkar, Kumar A. Microfilaria in fine needle aspiration smears of neoplastic lesions: A case series. Trop J Path Micro. 2017; 3:62-66.

14. Agarwal PK, Srivastava AN, Agarwal N. Microfialria in association with neoplasms. A report of six cases. Acta Cytol. 1982; 26:480-490.

15. Kolte SS, SatarkarRN, Mane PM. Microfilaria concomitant with metastatic deposits of adenocarcinoma in lymph node fine needle aspiration cytology: A chance finding. J Cytol. 2010; 27:78-80.

16. Jha A, Shrestha R, Aryal G, Pant AD, Adhikari RC, Sayami G. Cytological diagnosis of bancroftian filariasis in lesions clinically anticipated as neoplastic. Nepal Med Coll J. 2008; 10:108-114.

17. Pantola C, Kala S, Agarwal A, Khan L. Microfilaria in cytological smears at rare sites coexisting with unusual pathology: A series of seven cases. Trop Parasitol. 2012; 2:61-63.

18. HoeraufA. Filariasis: New drugs and new opportunities for lymphatic filariasis and onchocerciasis. Curr Opin Infect Dis. 2008; 21:673-681.

19. Hoerauf A, Mand S, Fischer K, Kruppa T, MarfoDebrekyei Y, DebrahAY, Pfarr KM, Adjei O, Büttner DW. Doxycycline as a novel strategy against bancroftian filariasis-depletion of Wolbachia endosymbionts from Wuchereria bancrofti and stop of microfilaria production. Med Microbiol Immunol. 2003; 192:211-216.

20. Thomsen, EK, Sanuku, N, Baea, Satofan S, Maki E, Lombore B, Schmidt MS, Siba PM, Weil GJ, Kazura JW, Fleckenstein LL, King CL. Efficacy, safety, and pharmacokinetics of coadministered diethylcarbamazine, albendazole, and ivermectin for treatment of bancroftian filariasis. Clin Infect Dis. 2016; 62:334-341.

(Received May 16, 2018; Revised August 22, 2018; Accepted August 25, 2018) 